\title{
Application of Negative Pressure Manual Dressing Change Combined with Mupirocin Ointment in the Treatment of a Case of a Patient with Cirrhosis Complicated with Deep Thigh Abscess
}

\author{
Yingying Meng',2, Wali Lukman Ahmed ${ }^{3}$, Jian Luo $^{4 *}$ \\ ${ }^{1}$ Medical School of Yangtze University, Jinzhou, China \\ ${ }^{2}$ Department of Gastroenterology, the Affiliated Hospital of Xuzhou Medical University, Xuzhou, China \\ ${ }^{3}$ Xuzhou Medical University, Xuzhou, China \\ ${ }^{4}$ Nursing Department, Union Hospital Affiliated with Tongji Medical College of Huazhong University of Science and Technology, \\ Wuhan, China \\ Email: *ljwhhbcn@163.com
}

How to cite this paper: Meng, Y.Y., Ahmed, W.L. and Luo, J. (2020) Application of Negative Pressure Manual Dressing Change Combined with Mupirocin Ointment in the Treatment of a Case of a Patient with Cirrhosis Complicated with Deep Thigh Abscess. Journal of Biosciences and Medicines, 8, 113-121.

https://doi.org/10.4236/jbm.2020.85011

Received: March 13, 2020

Accepted: May 5, 2020

Published: May 8, 2020

Copyright ( 2020 by author(s) and Scientific Research Publishing Inc. This work is licensed under the Creative Commons Attribution International License (CC BY 4.0).

http://creativecommons.org/licenses/by/4.0/

(c) (i) Open Access

\begin{abstract}
Objective: To explore the treatment and nursing characteristics for pressure injury on the inside of the thigh caused by forced posture in patients with cirrhosis and diabetes mellitus. Methods: A patient with cirrhosis and diabetes mellitus complicated with abscess of right inner thigh pressure injury was selected as the study object, who was admitted to Xuzhou Medical University Hospital on June 29, 2019. The analysis of the clinical diagnosis, treatment and nursing process were applied to explore the prognosis of the patient with cirrhosis and diabetes mellitus complicated with deep abscess of the thigh. Results: The traditional treatment including incision and drainage was not allowed, as the general treatment was not effective for the patients with cirrhosis and diabetes mellitus complicated with deep abscess in the inner thigh. After 8 days of negative pressure manual dressing change combined with mupirocin ointment treatment, the local skin condition returned to normal. Conclusion: After literature search, it was found that the above-mentioned cirrhosis combined with diabetes and deep abscess cases in the inner thigh were rare clinically. When the traditional treatment of incision and drainage is limited, negative pressure manual dressing change combined with antibiotics is an effective method, which is worthy of clinical application and promotion.
\end{abstract}




\section{Keywords}

Cirrhosis, Diabetes Mellitus, Pressure Injury, Deep Tissue Abscess, Negative Pressure Dressing Change

\section{Introduction}

The liver is an important organ for the synthesis and storage of nutrients, and malnutrition is common in the occurrence and development of liver diseases. Malnutrition seriously weakens the capacity of liver reserve and regeneration, which is closely related to clinical prognosis. It is reported that about $75 \%-90 \%$ of cirrhosis patients are accompanied with malnutrition in varying degrees when cirrhosis develops to decompensation of liver function [1]. The changes in liver structure in patients with cirrhosis can reduce the extraction of insulin from liver, leading to elevated insulin level in the whole body. Hyperinsulinemia can lead to insulin resistance through reduction of insulin receptor, reducing the affinity of insulin receptor, and reducing the effectiveness of insulin receptor exposed on the surface of target cells, which increases the risk of diabetes [2]. In addition, diabetes is an independent risk factor for the occurrence and progression of liver fibrosis [3], and diabetes can make metabolic disorders, such as accelerating the breakdown of metabolism, affecting the synthesis of albumin, and causing negative nitrogen balance in patients, thus reducing their resistance to infection. In addition, high blood sugar is conducive to the growth of bacteria. Therefore, the patient with cirrhosis combined with diabetes is more likely to be complicated with infection, especially deep abscess. The clinical symptoms of this type of patients are not typical, which can easily lead to the diagnosis and treatment untimely, resulting in serious consequences [4].

\section{Case Presentation}

On June 29, 2019, a patient with decompensated cirrhosis complicated with diabetes mellitus complicated with leg abscess was treated in our hospital. The method of excreting pus using the principle of negative pressure drainage would be moiré star ointment into the pus cavity, combined with the application of whole-body antibiotics. Comparing with the traditional cut-out drain treatment method, this method is simple, less painful and rapid recovery. After obtaining the informed consent from the patient, it is reported as follows:

\subsection{Case Information}

Patients, male, 37 years old, due to "repeated bloating for half a year, aggravated for 1 week" to "B virus hepatitis after cirrhosis, decompensated cirrhosis, type 2 diabetes, the inside of the right thigh pressure injury "was hospitalized in our hospital, On June 29, 2019. This patient had a history of hepatitis B for 10 years, and the diabetes history for 8 months. Physical examination: hepatic facies, 
sclera icterus, shallow lymph nodes are not swollen, abdominal enlargement, abdominal wall tension was high, abdominal wall veins were not revealed, the whole abdomen had no obvious tenderness and rebound pain, the liver and spleen could be touched at the position of 1 horizontal finger under the chest ribs, shifting dullness $(+)$, severe pitting oedema of the lower limbs. The patient had to force the right side to lie, complaining of pain in the abdomen and inner right thigh, and the numerical rating scale (NRS) was 4; Child-Pugh liver function grade $\mathrm{C}$. Laboratory evaluation showed that the patient had poor nutritional status and coagulation function, and had infection (Table 1).

\subsection{General Treatment}

On June 30,2019, the right inner thigh surface of the patient was red and swollen, with an area of about $5 \mathrm{~cm} \times 8 \mathrm{~cm}$. The local skin temperature increased, and numerical rating scale (NRS) was 5. Although local unstageable pressure injury was considered, thrombosis could not be excluded. The patient was given color doppler examination of the arteries and veins of both legs to eliminate thrombosis.

Table 1. Laboratory data.

\begin{tabular}{|c|c|c|}
\hline Project name & datas & units \\
\hline random blood sugar & 8.2 & $\mathrm{mmol} / \mathrm{l}$ \\
\hline red blood cell count & 1.9 & $10^{12}$ \\
\hline white blood cell count & 12 & $10^{9}$ \\
\hline haemoglobin & 67 & $\mathrm{~g} / 1$ \\
\hline total cholesterol & 1.96 & $\mathrm{mmol} / \mathrm{l}$ \\
\hline triglyceride & 0.47 & $\mathrm{mmol} / \mathrm{l}$ \\
\hline total protein & 50.9 & $\mathrm{~g} / \mathrm{l}$ \\
\hline albumin & 21.4 & $g / 1$ \\
\hline globulin & 29.5 & $\mathrm{~g} / \mathrm{l}$ \\
\hline pre-albumin & 0.04 & $\mathrm{~g} / \mathrm{l}$ \\
\hline urea & 11.38 & $\mathrm{mmol} / \mathrm{l}$ \\
\hline total bilirubin & 51.6 & $\mathrm{mmol} / \mathrm{l}$ \\
\hline direct bilirubin & 24.4 & $\mathrm{mmol} / \mathrm{l}$ \\
\hline blood potassium & 3.51 & $\mathrm{mmol} / \mathrm{l}$ \\
\hline blood sodium & 133.6 & $\mathrm{mmol} / \mathrm{l}$ \\
\hline alanine aminotransferase & 18 & $\mathrm{u} / \mathrm{l}$ \\
\hline aspartate aminotransferase & 57 & $\mathrm{u} / \mathrm{l}$ \\
\hline glutamyl transferase & 58 & $\mathrm{u} / \mathrm{l}$ \\
\hline antithrombins-III activity & 29 & $\%$ \\
\hline prothrombin time & 16.3 & $-\sec$ \\
\hline fibrinogen & 2.79 & $\mathrm{~g} / \mathrm{l}$ \\
\hline fibrinogen degradation products & 34.9 & $\mathrm{mg} / \mathrm{l}$ \\
\hline D-dimer & $>10$ & $\mathrm{ug} / \mathrm{ml}$ \\
\hline
\end{tabular}


The National Pressure Ulcer Advisory Panel (NPUAP) pointed out in the clinical practice guide issued in 2014 that the frequency of changing body position should be determined, according to the differences between individual [5]. Thus, during the period when the patient was emotionally stable and willing to cooperate, the posture should be changed every 0.5 - 2 hours, and a decompression pad was placed between the legs in the lateral position. The local pain of the patient was getting worse and the swelling was more obvious. The swelling area gradually expanded to $10 \mathrm{~cm} \times 10 \mathrm{~cm}$ on July 3, 2019. From July 10, 2019 to July 15,2019 , three purulent spots gradually formed on the skin surface, and the area of purulent spots gradually increased, the largest of which was about $0.5 \mathrm{~cm} \times$ $0.5 \mathrm{~cm}$. Under the doctor's order, the injured part was coated with erythromycin ointment, and cefoxitin was given by intravenous drip, but the effect was not good. On July 19, 2019, the surface of the abscess broke, and there was a light red liquid turbidity flowing out of the broken part, with oil floating on the surface. The exudate was put under the microscopic examination, then a large number of fat drops could be seen. Staphylococcus aureus was observed in the exudate bacterial culture, and drug sensitivity assay suggested its sensitivity to cefodizime. B-ultrasound indicated that there was a dark area of liquid. Considering the patient complicated with type 2 diabetes mellitus, who had general nutritional status and poor coagulation function, the plan at this point was to perform a conservative treatment. Otherwise if incision or puncture drainage was performed, there was a risk that the incision would not heal and the ulceration area enlarged. It was recommended that the patient should be treated daily with local pus drainage and given anti-infective treatment with cefodizime. Because the patient pain was severe, on the same day, according to the doctor's instructions, we gave the patient bucinnazine hydrochloride to relief pain and fructose $250 \mathrm{ml}$, cephalosporine $2.0 \mathrm{~g}$ IV bid to anti infection. After draining pus by hand, the local skin was covered with gauze soaked with iodophors, 20 min bid. The amount of pus and liquefying fat from the local ulceration site increased, and the local symptoms could not be relieved. The temperature of the patient rose to $38.8^{\circ} \mathrm{C}$, on July 21, 2019. Staphylococcus aureus was found in blood culture. On the same day, the patient was gave antipyretics and painkillers to control symptoms. According to the patient's condition, we analyzed the causes and discussed the new treatment and nursing plan.

\section{Cause Analysis}

1) The patient was in the period of decompensated cirrhosis and combined with low proteinemia, who's lower extremities were edematous, in a state of poor overall nutritional.

2) The patient must be forced to lie on the right side to relieve dyspnoea, because of a lot of hydrothorax and ascites, which resulted in long-term compression of the inner side of his right thigh and tissue hypoxia.

3) At the same time, the patient was complicated with diabetes. Diabetes can reduce the immunity of the body. Hyperglycaemia can promote the rapid growth 
of bacteria in the tissue and accelerate the formation of abscess. Because the location of abscess was deep, the effect of local surface coating with antibiotics treatment was not good.

4) The patient had poor compliance, irritability, and did not cooperate with nursing work, such as refusing to cooperate to turn over, etc. He began to abandon himself, due to the tense relationship with his family and lacking of company.

5) The site of abscess in patients was rare in clinical work, which had not been paid enough attention by medical staff.

\section{Local Application of Mupirocin Ointment by the Principle of Negative Pressure Suction and Nursing Effect}

After local external used of iodophor and mupirocin ointment, the effect was not good, and the patient's local condition was not alleviated. On 2019-07-22, considering the deep infection site of the patient, with the doctor's consent, we used the principle of negative pressure suction to change the dressing in order to increase the contact between the drug and the infected surface. The specific operation steps are as follows: The patient laid on the right side, and a disposable absorbent towel sheet was placed under the right thigh, and the local skin was disinfected with Iodophor cotton ball. The nurse wore sterile gloves to squeeze out pus and liquefied fat, and pressed on the skin with both hands. At the same time, assistant with cotton swab took some mupirocin ointment like ball slightly larger than the broken surface, placed it on the broken surface, then, removed the palm, ointment was inhaled into the pus cavity. In this way, the mupirocin ointment was inhaled into the other two pus cavities. A proper amount of mupirocin ointment was applied to the whole swelling surface. After the ointment was liquefied, the nurse wearing sterile gloves massaged the local skin gently, the drug was changed in this way for every 8 hours. During two dressing changes, local skin was covered with gauze soaked with iodophor for $20 \mathrm{~min}$. The patient's position was changed $\mathrm{q} 2 \mathrm{~h}$, and a decompression pad was placed between his knees. The next day, the pain and swelling subsided, the amount of excretion of pus and liquefied fat decreased day by day. After 4 days, the ulcer surface healed, and the symptoms of redness, swelling, heat and pain disappeared, the local skin was harder than the surrounding skin. Numerical rating scale (VAS) 0. After the 8th day, the local skin tended to be normal, but was slightly harder than the surrounding skin.

\section{Vigilance of Addiction to Narcotic Drugs and Psychological Intervention}

Pressure injury can cause severe physical pain, affect the quality of life of individuals and their families, increase medical costs and hospital stays. Because of the patient's grumpy personality and domestic violence, it was still the same during the hospitalization, which leads to the situation that his family were not willing to go to the hospital to accompany and causes a lot of inconvenience to 
the medical treatment and nursing. Due to the lack of family care, the patient's mood was negative, indifferent and poor compliance. As local swelling become more pronounced, the pain was severe, and seriously affected his sleep. According to the doctor's instructions, bucinnazine hydrochloride was given muscle-filled to relieve pain with good effect.The patient's demand for analgesic was more and more frequently, suggesting that there was a tendency to exaggerate pain.

In view of the psychological state of the patient, the doctor contacted a psychiatrist to help the patient eliminate the gap with their families, and explained the treatment and nursing plan to him and his family to get the cooperation of the patient and his family. The mechanism and addictive ability of the narcotic drugs were explained to the patient to alleviate the patient's dependence on the drug.

\section{General Nursing Measures}

\subsection{Assessment of the Nutritional Status}

The patient's BMI was $24.5 \mathrm{~kg} / \mathrm{m}^{2}$, within the normal range. Body mass index (BMI) is widely used in clinical practice, is a reliable parameter for detecting malnutrition. However, the patient with liver cirrhosis was in decompensation stage with ascites and water-sodium retention. At this time BMI cannot accurately respond to the patient's protein-thermal nutrition. The Royal Free Hospital Nutritional Prioritizing Tool (RFH-NPT) is suitable for patients with liver disease by means of simple and easy-to-answer clinical questions including complications of body fluid storage, eating, weight changes, etc. [1]. The patient's RFH-NPT: 5 score, in high risk of malnutrition.

\subsection{Develop Nutrition Plan}

If the nutritional support measures are not taken actively in the patients with innutrition, the adverse factors such as the reduction of protein anabolism, muscle atrophy and subcutaneous fat decrease will lead to the formation of pressure injury. In addition, the patient with diabetes, if given a high calorie diet, blood sugar will be difficult to control, if given a high protein diet, there is a risk of inducing hepatic encephalopathy. Therefore, the nutrition, diabetes and digestive department specialist nurses were invited to formulate diet recipes according to the European Society of Clinical Nutrition and Metabolism (ESPEN) guidelines issued in 2006 [6], with the aim to control the total calorie intake and adjust in time according to the patient's condition.

1) The patients with hydrothorax and ascites must limit the intake of water and sodium. The total intake of water was about $1000 \mathrm{ml} / \mathrm{d}$, and the intake of sodium was less than $2 \mathrm{~g} / \mathrm{d}$. Sausage, pickles, biscuits and other foods with high sodium content were forbidden.

2) Malnutrition is a risk factor for pressure injury, and patients with hypoproteinaemia are more likely to occur [7]. Protein is the material basis for hepatocyte repair and maintenance of plasma colloid osmotic pressure. When hepatic 
encephalopathy did not occur, it was recommended that protein intake accounts for $15 \%-20 \%$ of total calories, $1.2-1.5 \mathrm{kcal} / \mathrm{kg} / \mathrm{d}$. When blood ammonia was normal, the patient chose high-quality animal protein, such as: fish, chicken, eggs, soy products, lean pork etc. When the blood ammonia rised, protein was limited or prohibited, according to the patient's condition. Plant protein, such as beans, walnuts, almonds and their products were also suitable for gradually increasing the protein intake after the condition improved.

3) Because the glycogen synthesis and storage function of patients with cirrhosis of the liver is impaired, it makes the patient more susceptible to hypoglycaemia [8]. Although the patient had diabetes, carbohydrate intake was not restricted to prevent hypoglycaemia. Carbohydrate intake accounts for $45 \%-65 \%$ of total calories, with recommended intakes of $25-35 \mathrm{kcal} / \mathrm{kg} / \mathrm{d}$, without hepatic encephalopathy; $35 \mathrm{kcal} / \mathrm{kg} / \mathrm{d}$ with hepatic encephalopathy. Snacks can be eaten at 4 - 6 meals per day to reduce the risk of hypoglycaemia [9]. Non-rough foods with low glycemic index were beneficial. In order to increase appetite, we chosed non-nutritional sweeteners, such as xylitol, protein sugar, stevia tablets, etc for the patient and monitored the patient's blood sugar level before and after meals.

4) The total cholesterol is involved in the composition of cells and cell membranes, and it plays an important role in the permeability of the biofilm, the insulation of the nerve myelin and the protection of cells from some toxins, which is of great significance to hepatitis B. Because the patient's total cholesterol level was low, so high cholesterol foods did not have to be strictly restricted [10]. The fat intake in principle did not exceed $30 \%$ of the total calories and saturated fatty acids were $<7 \%$.

5) Vitamins play an important role in material metabolism. Although they are required in small quantities, they must always be supplied by food. Because the patient's blood sugar was well controlled, his diet was reduced from 6 meals to 3 meals per day, divided by $1 / 3$. Fruits and vegetables, such as watermelon, apple and tomato, were added between the two meals. They could be juiced to drink for several times. Besides, tender vegetables should be consumed appropriately.

6) The patient's wall of the oesophageal and gastric vein was weak and lack of elastic contraction, which could easily lead to massive haemorrhage. All food must be presented as soft food, the vegetables need to be fresh and tender, meat should not be mixed with broken bones and crustaceans, grains should not be mixed with chaff. The patients should avoid eating raw, cold, hard, hot, spicy foods and condiments, and chew slowly while eating.

7) Intravenous nutritional support was used, including human albumin, plasma, branched-chain amino acid to improve the nutritional status of the patient.

\section{Ward Management}

Keepping the room temperature at $20^{\circ} \mathrm{C}-22^{\circ} \mathrm{C}$, relative humidity at $50 \%-60 \%$, indoor ventilation twice a day. UV disinfection was needed. If contaminated with pus, the bed linen need to be replaced timely and keep flat and dry. 


\section{Discussion}

Mupirocin ointment is a kind of white hydrophilic external antibiotic ointment with strong antibacterial activity, the local absorption less than $1 \%$, which is almost non-toxic to human body [11]. In recent years, mupirocin ointment has been widely used in wound, which can quickly and effectively control the infection of the wound. However, there are few reports on the application of deep skin infection. Treatment and nursing methods were mainly based on puncture drainage, cut drainage or persistent negative pressure attraction, which leaded to a long treatment cycle, great pain and high cost for patients. In this case, the patient was in the decompensated stage of cirrhosis with diabetes mellitus [12] [13] [14]. The resistance of the patient to bacteria was very poor and easy to get infected. In addition, the patient had a large amount of ascites and was forced to lie on the right side. The inner side of his right thigh was the compression surface, which was not a common part of pressure injury. It was so easy to be ignored in nursing work. Moreover, the subcutaneous fat in this part was rich. Long term compression could easyly cause fat liquefaction and aseptic necrosis.Pressure injury can occur in any part of the body under pressure [15]. When the patient was complicated with circulatory disorders, coagulopathy and a variety of basic diseases, local infection could easily occur. If the formed abscess was not treated in a timely and reasonable manner, septicaemia may occur, and even life-threatening. When we encounter this situation, the application of abscess incision and drainage method is limited, the local external application of antibiotics can not achieve good results, and the cost of using closed negative pressure suction device is high, we can try this method.

\section{Conclusion}

The negative pressure manual dressing change has the characteristics of no trauma, less pain, low cost, simple operation, and it can fully lead the medicine into the purulent cavity, increase the drug contact area, effectively control the local infection, which is very beneficial to improve the prognosis of the disease.

\section{Conflicts of Interest}

The authors declare no conflicts of interest regarding the publication of this paper.

\section{References}

[1] Palmer, L.B., Kuftinec, G., Pearlman, M., et al. (2019) Nutrition in Cirrhosis. Current Gastroenterology Reports, 21, 38-48. https://doi.org/10.1007/s11894-019-0706-5

[2] Shanik, M.H., Xu, Y., Skrha, J., et al. (2008) Insulin Resistance and Hyperinsulinemia: Is Hyperinsulinemia the Cart or the Horse? Diabetes Care, 31, S262-S268. https://doi.org/10.2337/dc08-s264

[3] Elkrief, L., Rautou, P.-E., Sarin, S., et al. (2016) Diabetes Mellitus in Patients with Cirrhosis: Clinical Implications and Management. Liver International, 36, 936-948. https://doi.org/10.1111/liv.13115 
[4] Kim, E.J., Ha, K.H., Kim, D.J., et al. (2019) Diabetes and the Risk of Infection: A National Cohort Study. Diabetes \& Metabolism Journal, 43, 804-814. https://doi.org/10.4093/dmj.2019.0071

[5] Haesler, E., Kottner, J. and Cuddigan, J. (2017) The 2014 International Guideline Development Group. The 2014 International Pressure Ulcer Guideline: Methods and Development. Journal of Advanced Nursing, 73, 1515-1530. https://doi.org/10.1111/jan.13241

[6] Plauth,M., Bernal, W., Dasarathy, S., et al. (2019) ESPEN Guideline on Clinical Nutrition in Liver Disease. Clinical Nutrition, 38, 485-521. https://doi.org/10.1016/j.clnu.2018.12.022

[7] Neloska. L., Damevska, K., Nikolchev, A., et al. (2016) The Association between Malnutrition and Pressure Ulcers in Elderly in Long-Term Care Facility. Open Access Macedonian Journal of Medical Sciences, 4, 423-427. https://doi.org/10.3889/oamjms.2016.094

[8] Hamed, A.E., Elsahar, M., Elwan, N.M., et al. (2018) Managing Diabetes and Liver Disease Association. Arab Journal of Gastroenterology, 19, 166-179. https://doi.org/10.1016/j.ajg.2018.08.003

[9] Nakaya, Y., Okita, K., Suzuki, K., et al. (2007) BCAA-Enriched Snack Improves Nutritional State of Cirrhosis. Nutrition, 23, 113-120. https://doi.org/10.1016/j.nut.2006.10.008

[10] Krautbauer, S., Wiest, R., Liebisch. G., et al. (2017) Associations of Systemic Sphingolipids with Measures of Hepatic Function in Liver Cirrhosis Are Related to Cholesterol. Prostaglandins \& Other Lipid Mediators, 131, 25-32. https://doi.org/10.1016/j.prostaglandins.2017.06.004

[11] Vaughn C. (2012) Drugs and Lactation Database: LactMed. Journal of Electronic Resources in Medical Libraries, 9, 272-277. https://doi.org/10.1080/15424065.2012.735134

[12] Hasan, M.Y., Teo, R. and Nather, A. (2015) Negative-Pressure Wound Therapy for Management of Diabetic Foot Wounds: A Review of the Mechanism of Action, Clinical Applications, and Recent Developments. Diabetic Foot \& Ankle, 6, 27618. https://doi.org/10.3402/dfa.v6.27618

[13] Liu, F., Zhao, G.P., Qin, H.X., et al. (2018) Influence of 3 Different Negative Pressure Values in Vacuum Sealing Drainage on Curative Effect of Stage III and IV Pressure Ulcer. Chinese Nursing Research, 32, 123-124.

[14] Zhou, C.-Y. and Hu, X.F. (2018) Ultrasound Guided Abdominal Abscess Puncture and Surgical Incision for Drainage in the Application of Patients with Abdominal Abscess. China Modern Medicine, 25, 42-44.

[15] Wang, S.Y. (2012) Nursing Care of Two Cases with Special Parts of Pressure Ulcer. Chinese Nursing Research, 26, 192. 